\title{
Improving the attendance for new psychiatric out-patient referrals
}

\author{
Martin Baggaley, Honorary Senior Registrar, Munro Clinic, Guy's Hospital, \\ London Bridge, London SE1 9RT
}

A significant proportion of patients referred to psychiatric out-patient clinics fail to attend the first appointment. Previous work suggested that typically $30 \%$ do not attend (Burgess \& Harrington, 1964; Skuse, 1975) and this results in an inefficient utilisation of psychiatrists' time. One solution would be to overbook new patients by $30 \%$. Unfortunately the proportion of non-attendance at each clinic is variable and difficult to estimate in advance. Psychiatrists have been shown to be poor predictors of non-attendance from examining referral letters (Woods, 1992). Overbooking might result in a long waiting time and short time for assessment. Previous research has suggested patients are more likely to attend if they received a personal letter rather than a standard card, had a previous psychiatric history, and if they were seen in a health centre rather than a hospital (Hillis, 1990). Allaying the patient's fears and misconceptions about psychiatry also improved attendance (Skuse, 1975). Work in a child and family setting suggested that sending a family questionnaire prior to the appointment reduced the non-attendance rate (Mathai \& Markantonakis, 1990).

The aim of this study was to see if first attendance to a general adult psychiatric out-patient clinic could be improved by asking the referred patient to confirm they actually wanted an appointment, either in writing or by phone, before an appointment was sent.

\section{The study}

This was a prospective study of all new referrals to an out-patient clinic at a London teaching hospital run by two consultants in general adult psychiatry. Referrals who had been seen previously by the psychiatric department at the hospital were excluded. The study lasted until 100 consecutive patients had been collected, which took approximately 20 weeks. Referrals were divided at random by the clinic secretaries into two groups. One group (N) were sent appointments in the normal way, (a brief pre-printed letter with the details filled in together with directions to the clinic). The second group (Y) were sent a letter explaining that they had been referred to the clinic and asking them to telephone or return an enclosed form if they wished to be seen. They were also asked to contact the clinic if they did not want to attend. Once they had contacted the clinic they were sent an appointment. Patients in group Y were allocated appointment times depending on the date of the referral rather than the date of reply to assure approximately equal lengths of time between referral and appointments in both groups.

\section{Findings}

A total of 103 patients were included in the study of whom 54 were sent appointments in the normal way (group N) and 49 were asked to request an appointment (group Y). The mean intervals between referral date and appointment were 51 days in group $\mathrm{N}$ and 47 days in group $Y$. In group N, $15(28 \%)$ did not attend for their appointment. In group Y, $14(29 \%)$ did not reply and $4(8 \%)$ replied but indicated they did not want an appointment. A total of 31 requested an appointment and of these $30(97 \%)$ turned up. The rate of non attendance between the two groups after being given an appointment differed significantly $(P<0.05)$.

The two groups did not differ significantly on age, sex or delay between date of referral and date of appointment allocated. Diagnostic information from the initial referral letter suggested that the type of patient who did not attend from group $\mathrm{N}$ was similar to that who failed to reply in group Y. Fifty-three per cent of group $N$ who failed to attend and $64 \%$ of group $Y$ who did not reply were considered by the GP to be suffering from alcohol problems, social problems, had committed deliberate self harm or had a personality disorder. These comprised $23 \%$ of referrals but $58 \%$ of non replies or DNAs (did not attend). Similarly referrals from non GPs (psychiatrists, surgeons, gynaecologists, the accident and emergency department and physicians) resulted in a high rate of non attendance or failure to reply. These comprised $20 \%$ of referrals but $52 \%$ of non-replies or DNAs.

The four patients in group $Y$ who replied but declined an appointment were all suffering from depression according to the referral letter.

\section{Comment}

Asking patients to request an out-patient appointment resulted in a more efficient service in that of those allocated an appointment $97 \%$ turned up. This 
prevents psychiatrists wasting valuable time which could be spent seeing other patients. In this study almost $30 \%$ of new appointments were wasted. However the percentage of patients referred that are actually seen in out-patient clinics does not improve. Patients who do not attend appear to be the same patients who do not request an appointment. Such individuals are found more often in particular diagnostic groups and from particular patterns of referral but unfortunately cannot be predicted with certainty. It may be that they do not need specialised psychiatric assessment at all or if they do an ordinary psychiatric out-patient appointment is not the way to reach them.

It is suggested one way to improve attendance at out-patient clinics is to ask patients to say if they want an appointment. This leaves a significant proportion of patients whom a referring agency asks to be seen but who do not reply or do not attend if sent an appointment; an alternative method of service provision is needed for this group.

\section{Acknowledgements}

I wish to thank the hard working receptionists at the Munro Clinic Guy's for their valuable help and to Professor J. P. Watson for his comments and advice.

\section{References}

Burgess, J. \& HaRrington, J. (1964) 200 psychiatric outpatient non-attenders. Case Conference, 11, 58-60.

HiLlis, G. (1990) Rejection of psychiatric treatment. Psychiatric Bulletin, 14, 149-150.

MatHAI, J. \& MarkantonaKIS, A. (1990) Improving initial attendance to a child and family psychiatric clinic. Psychiatric Bulletin, 14, 151-152.

SKuSE, D. (1975) Attitudes to the psychiatric out-patient clinic. British Medical Journal, 3, 469-471.

Woods, J. (1992) Can psychiatrists predict which new referrals will fail to attend? Psychiatric Bulletin, 16, $18-19$.

\title{
Assisted suicide or culpable suicide: is there a difference?
}

\author{
Hari D. Maharajh, Consultant Psychiatrist, St Ann's Hospital, Port of Spain, \\ Trinidad
}

In 1991, Derek Humphry published Final Exit - The Practicalities of Self-Deliverance and Assisted Suicide for the Dying. The book was written for mature adults who were suffering from terminal illnesses and required guidance in committing suicide, but it has encouraged suggestive and susceptible people to attempt suicide (Lavin et al, 1992). There appears to be a thin line between assisted suicide and culpable suicide. 'Culpable suicide' is used here to describe contributory negligence attributed to persons who unknowingly assist another person's suicide.

Trinidad and Tobago has a population of 1.3 million, of whom $43 \%$ are Indians. Most Indians are Hindus, comprising approximately $30 \%$ of the total population. The suicide rate is highest among Indians, and paraquat poisoning is the main cause of death. Legislation to restrict the availability of paraquat (a herbicide) has never been implemented. This substance, therefore, is readily available in the agricultural regions, where most Indians live.
Theories put forward for the high rate of suicide among Indians in Trinidad include marginalisation and ethnic disadvantage (Parasram, 1992), stress, the high rate of unemployment, family discord, and an increase in reporting of suicides by the media (Maharajh, 1992). Religion and culture appear also to contribute.

Two cases of culturally determined culpable suicide are presented.

\section{Case 1}

An 18-year-old female student from an orthodox Hindu family was admitted to the medical ward of the general hospital after swallowing paraquat. She died two weeks later. At interview she had revealed that her father had arranged a marriage for her of which she had disapproved. When she attempted to leave home to visit her boyfriend of another religious persuasion, her father insisted that the only way she was going to leave his house that day was in a box. That night she drank the paraquat. 